

\title{
INNOVACIÓN TECNOLÓGICA EN LA RESOLUCIÓN DE PROBLEMÁTICAS SOCIO- PRODUCTIVAS LOCALES. CASO DE ESTUDIO: CONCORDIA, ENTRE RÍOS-ARGENTINA
}

\author{
TECHNOLOGICAL INNOVATION IN THE RESOLUTION OF LOCAL \\ SOCIO-PRODUCTIVE PROBLEMS. CASE STUDY: CONCORDIA, ENTRE \\ RÍOS, ARGENTINA
}

\author{
VALERIA FENOGLIO \\ Doctora en Arquitectura, Investigadora \\ Universidad Nacional de Córdoba (UNC) - Centro de \\ Investigaciones y Estudios sobre Cultura y Sociedad (CIECS) \\ -Consejo Nacional de Investigaciones Científicas y Técnicas \\ (CONICET), Córdoba, Argentina \\ https://orcid.org/0000-0003-0243-1798 \\ valefenoglio3@gmail.com
}

\begin{abstract}
RESUMEN
El presente trabajo pone en relevancia la promoción del diseño y gestión de innovaciones tecnológicas para la resolución de problemáticas socio-productivas locales. Desde una visión de innovación, que intenta superar la óptica exclusivamente económica, se toma como caso paradigmático una experiencia de producción de hábitat desarrollada en la localidad de Concordia (Entre Ríos, Argentina). La misma, desarrolla colectivamente una tecnología asociada a sistemas constructivos en madera, con el fin de promover procesos productivos sustentables a partir de recursos y saberes locales. Asimismo, propone una innovación que intenta modificar los modos productivos actuales, superando la transferencia tecnológica unidireccional, por nuevas formas de resolución de problemas de manera cooperativa y solidaria. Mediante un estudio interpretativo, el trabajo tiene por objetivo principal analizar el caso desde la perspectiva crítica de la tecnología, relacionando el concepto de código técnico (normas cognitivas y sociales que configuran los procesos de producción de conocimiento) con los modos de diseñar y gestionar la producción de hábitat en la experiencia. Como resultado, se dilucidan aspectos emergentes del caso que, a modo de insumos o lineamientos, permiten contribuir a la generación de nuevos abordajes para la producción de un hábitat sustentable y más justo cognitivamente.
\end{abstract}

\section{Palabras clave}

Energía incorporada, emisiones de CO2, residuos de demolición y construcción, diseño por ordenador

\section{ABSTRACT}

This paper highlights the promotion of the design and management of technological innovations for the resolution of local socio-productive problems. Working from a point of view on innovation that attempts to overcome an exclusively economic perspective, a habitat production experience carried out in the town of Concordia (Entre Ríos, Argentina) was selected as a paradigmatic case. This experience collectively develops a technology related to wood construction systems in order to promote sustainable production processes based on local resources and knowledge. Likewise, it proposes an innovation that attempts to modify the current modes of production and surpass unidirectional technological transfer with new ways of solving problems cooperatively and charitably. By means of an interpretive study, the main objective is to analyze the case from the critical perspective of technology, relating the concept of technical codes (cognitive and social norms that configure the processes of knowledge production) with the ways of designing and managing habitat production in the experience. As a result, emerging aspects of the case are elucidated, which in the form of inputs or guidelines make it possible to contribute to the creation of new approaches for the production of a sustainable habitat that is more cognitively just. 


\section{INTRODUCCIÓN}

\section{PRESENTACIÓN DEL PROBLEMA}

La búsqueda de nuevos abordajes para la producción de un hábitat sustentable y más justo cognitivamente es el principal motor de marcha en el presente trabajo. Desde hace varias décadas, los Estudios Sociales de la Ciencia y la Tecnología (ESCyT) intentan cuestionar el papel del conocimiento científico-tecnológico en relación con la sociedad. Entre las diferentes disciplinas y perspectivas teóricas que integran estos estudios subyace una crítica común: superar la visión clásica y determinista de la Ciencia y la Tecnología (CyT), donde se prioriza el producto por sobre cualquier otra racionalidad, suponiendo que todo desarrollo tecnológico es la solución a un problema existente sin considerar contextos ni actores particularizados. Autores como Dagnino (2008) consideran que esta concepción lleva a que sea un asunto técnico y no político, lo cual ejemplifica a partir de una especie de barrera virtual que se forma entre el ambiente de producción científica-tecnológico y el contexto social, político y económico de nuestras sociedades. En América Latina, cuestiones como la democratización del conocimiento y la desigualdad social vienen siendo abordadas desde esta perspectiva teórica por diversas instituciones e investigaciones, cuyo énfasis está puesto en generar un manejo más apropiado de CyT.

En Argentina, todavía falta un largo camino en la construcción de abordajes alternativos que generen procesos sustentables en el campo del hábitat. Pero desde hace algunos años, un grupo de investigadores, del Consejo Nacional de Investigaciones Científicas y Técnicas de la Nación (CONICET), viene desarrollando procesos de innovación tecnológica en distintas partes de Argentina. La convicción ideológica de dichos procesos es el fortalecimiento de emprendimientos pequeños, ya que estos contribuyen a la generación de empleo (70\% del empleo productivo está en manos de dichos emprendimientos) y la distribución de la renta que este tipo de proceso económico promueve, al fomentar articulaciones comerciales para un abastecimiento y consumo dentro del país (Peyloubet, 2018).

Articulando la problemática del hábitat con los procesos productivos, el equipo de investigación ha desarrollado innovaciones tecnológicas con madera de cultivo local, considerando el alto potencial socio-productivo que este tiene. Según Diana Guillén, las características de Argentina ubican a este país entre las regiones del mundo con mayores ventajas naturales por el rápido crecimiento de sus plantaciones y su potencial productivo (citado en Servicio Nacional de Sanidad y Calidad Agroalimentaria, 2014).

La ciudad de Concordia en la provincia de Entre Ríos (estudio de caso) cuenta con la mayor superficie boscosa de Eucalyptus Grandis. Como problemática local, se advierte que el destino principal de esta producción es el aserrado (45\%), predominando las pequeñas industrias que utilizan madera corta y producen embalajes, pallets y cajones. El porcentaje restante (55\%) se considera subproducto: aserrín, costaneros, corteza y refilados destinados a la fabricación de tableros de aglomerados. En ese escenario, no se identifican procesos locales que otorguen valor agregado y distribución de renta sobre aquel $45 \%$ restante.

Frente a esta problemática socio-productiva cabe preguntarse: ¿Cómo diseñar y gestionar propuestas tecnológicas que contribuyan al mejoramiento de territorios locales? ¿Cómo proceder a otras operaciones alternativas que den lugar a nuevos códigos o normas tecnológicas más adecuadas social y técnicamente?

Siguiendo a Albornoz (2013), la innovación está hoy en el centro de las políticas que los países de América Latina aplican para impulsar el desarrollo y la equidad. Sin embargo, hubo una traslación mimética de políticas creadas en economías en las que existe una fuerte demanda de nuevos conocimientos a contextos económicos en los que tal demanda es muy escasa. (Albornoz, 2013). Asimismo, se utiliza frecuentemente la innovación para incidir en la competitividad de las empresas y muy poco para relacionarla con la mejora de los problemas sociales (OEI, 2014).

Otro cuestionamiento que se le otorga a los procesos tecnológicos de innovación son las formas tradicionales con que se legitima y construye el conocimiento en dichos procesos. Para Santos (2009), existe un intento por desmontar la dicotomía existente entre conocimiento experto o científico y conocimiento local o popular, con el objetivo de rescatar los saberes que surgen de las propias experiencias sociales.

En ese marco, el trabajo tiene por objetivo principal comprender e interpretar las particularidades que fueron dadas en un proceso de desarrollo tecnológico, que vincula al sector de CyT con el territorio. Metodológicamente, se analiza el caso desde la perspectiva crítica de la tecnología, relacionando el concepto de código técnico (normas cognitivas y sociales que configuran los procesos de producción de conocimiento) con los modos de diseñar y gestionar la producción de hábitat en la experiencia. Para ello, se elaboraron una serie de presupuestos de partida y categorías analíticas ad hoc; estas últimas reconocidas en el presente trabajo como las premisas de diseño que el equipo de investigación emplea en su labor investigativa. En cuanto a los resultados, se dilucidaron aspectos emergentes del caso, que a modo de insumos o lineamientos pretenden contribuir a la generación de nuevos abordajes para la producción de un hábitat sustentable y más justo cognitivamente.

\section{MARCO TEÓRICO-CONCEPTUAL}

A lo largo de la historia, los procesos de industrialización y desarrollo tecnológico se han visto continuamente acompañados por contra-corrientes de innovación, como reacción y cuestionamiento a las trayectorias dominantes a partir del desarrollo de diferentes movimientos (denominados como Tecnología Apropiada y Tecnología Social), con formas 
de innovación alternativa y socialmente inclusiva (Fressoli, Smith, Thomas y Bortz, 2016).

Para el presente trabajo interesa destacar a la Tecnología Social como un movimiento de innovación contemporáneo que busca proveer un nuevo modo de desarrollar e implementar tecnologías (de Producto, Proceso y Gestión), orientadas a la generación de dinámicas de inclusión socioeconómica y desarrollo sustentable (Thomas y Becerra, 2014). Este movimiento aporta una dimensión procesual, una visión ideológica y un elemento de operacionalidad diferente que no se encuentra en las actuales tecnologías disponibles (Dagnino, 2008).

La integración de conceptos teóricos y concepciones ideológicas provenientes de diferentes enfoques disciplinarios (filosofía de la tecnología; sociología de la tecnología; economía del cambio tecnológico, etc.) constituyen el marco analítico-conceptual con el que se aborda el estudio de la Tecnología Social, partiendo de una revisión crítica de la llamada Tecnología Apropiada (TA), con el objetivo que, desde allí, se pueda construir otra tecnología de base cognitiva diferente.

La crítica señalada a esta tecnología (TA), originada en la década del 60 , se asienta sobre la idea de que la solución al problema se construye de una manera unidireccional, generando un juego de oferta y demanda, donde la lógica de transferencia subordina todo el proceso. Esto significa que se ha venido desarrollando una serie de tecnologías a modo de stock, utilizadas muchas veces según la demanda, y que principalmente ha dejado a un lado aquel conocimiento tradicional o tácito, que la academia no legitima. Así, se desperdicia el alto potencial de saberes que se encuentra contenido en actores y experiencias sociales, advirtiéndose que el conocimiento aún se construye de forma exclusiva, de arriba hacia abajo, a partir de un proceso unidireccional, es decir, a modo de transferencia.

Por lo tanto, consideramos que, en la búsqueda de alternativas para la producción de hábitat, la Tecnología Social representa un camino que va en esa dirección. Entre los modelos teóricos que conforman estos estudios, el Constructivismo Social y la Teoría Crítica de la Tecnología se constituyen en respuesta radical a la visión mono-dimensional, lineal y determinista de la tecnología. Respecto al Constructivismo Social, Valderrama (2004) describe esta teoría como una manera de abrir la caja negra del conocimiento para descubrir que, en su interior, se presentan dinámicas que se debe estudiar porque están íntimamente ligadas a procesos sociales. Esta teoría ofrece la posibilidad de considerar a la tecnología como una construcción social y como resultado de procesos de negociación y de interpretaciones diferentes entre grupos sociales relevantes, hasta que la tecnología llega a ser lo que finalmente es. Sin embargo, consideramos que no basta con abrir la caja negra e identificar los actores relevantes y sus intereses, sino que también es necesario que, previo al diseño de las tecnologías, operen mecanismos más democráticos. En ese sentido, la propuesta de Andrew Feenberg gira en torno a la extensión del valor de democracia al ámbito de la tecnología. El autor sugiere que la noción de racionalización, hoy centrada en la idea de progreso y eficiencia, debiera fundirse en la responsabilidad de la acción técnica por los contextos humanos y naturales. Para ello, Feenberg (2006) plantea cambiar los valores dominantes de la racionalidad tecnológica, incorporando a priori, en el diseño de la tecnología, aspectos sociales, culturales y ambientales alternativos, que propicien formas más participativas y democráticas. Asimismo, hace referencia a la noción de código técnico como a la realización de un interés bajo la forma de una solución técnicamente coherente a un problema. De esta manera, "el producto de las elecciones técnicas respalda el modo de vida de uno u otro grupo social influyente" (Giuliano, 2012, p. 2). En la mayoría de los casos, los intereses de grupos dominantes se materializan en códigos técnicos que, de manera invisible, se sedimentan en reglas, procedimientos, instrumentos y artefactos en pro de la búsqueda de poder y de ventajas por una hegemonía dominante. Para Feenberg, las características de este código técnico son autoritarias y verticalistas y acarrean problemas serios, incluso en los sectores más avanzados de la sociedad (Tula Molina y Giuliano, 2015). Este abordaje hace posible apreciar con mayor claridad que en los diseños tecnológicos aparecen beneficiados y damnificados, por lo que su concretización representa una serie de luchas y estrategias entre actores diversos para desarrollar una u otra alternativa tecnológica. Según Feenberg (2006), la tecnología puede constreñir y colonizar, pero también puede liberar potencialidades reprimidas del mundo de la vida que de otra forma hubieran quedado sumergidas. Por consiguiente, para este teórico, la tecnología es esencialmente ambivalente y disponible para varios tipos de desarrollo. Además, autores como Tula Molina y Giuliano (2017) sostienen que en el diseño de la tecnología intervienen otros aspectos tales como intereses, costumbres, valores y relaciones de poder, por lo que resulta necesario que sean puestos en discusión por parte de una pluralidad de actores sociales, no solo los expertos, y organizados en base a principios democráticos.

\section{METODOLOGÍA}

El presente trabajo se posiciona en el marco del paradigma Interpretativo", cuyo "fundamento radica en la necesidad de comprender el sentido de la acción social en el contexto del mundo de la vida y desde la perspectiva de los participantes" (Vasilachis, 2006, p. 48). Una investigación posicionada desde este paradigma considera que el caso en sí mismo desempeña un papel fundamental, que puede aportar a la comprensión del problema en estudio (Kazez, 2009). 
Siguiendo esa línea, el propósito de la investigación aquí expuesta es entender y dilucidar profundamente un caso particular de producción de hábitat (Concordia, Entre Ríos). En primer lugar, interesa abordar dicho caso desde la tradición cualitativa, principalmente por dos características puntuales: a) por lo que estudia esta tradición: contextos, procesos, sentidos, significados y relatos; y b) por la meta y su finalidad: búsqueda de lo nuevo y provisión de nuevas perspectivas sobre lo que se conoce, describe, explica, elucida, construye y descubre (Vasilachis, 2006).

En ese marco, se propone analizar el caso desde la perspectiva crítica de la tecnología, relacionando el concepto de código técnico con los modos de diseñar y gestionar la producción de hábitat en la experiencia. Así, se entiende "código técnico" como aquellas normas cognitivas y sociales que configuran los procesos de producción de conocimiento y cuyo resultado determina un tipo de innovación tecnológica. Este enfoque permite apreciar con claridad que en los diseños tecnológicos aparecen beneficiados y damnificados, por lo que su concretización representa una serie de luchas y estrategias entre actores diversos para desarrollar una u otra alternativa tecnológica. Desde esta visión crítica acerca de la tecnología, se enuncian una serie de presupuestos de partida que guiaron la actual reflexión:

- Los conocimientos locales se constituyen en insumos indispensables para producir transformaciones capaces de mejorar la calidad de vida de las personas, compatibilizándose con el contexto natural y social.

- En la mayoría de las innovaciones tecnológicas, dentro del campo del hábitat, no se incorpora el conocimiento tradicionalmente utilizado (saber local) y se privilegia al producto $\mathrm{u}$ artefacto tecnológico sobre los procesos o gestiones tecnológicas.

- La aplicación de modelos de gestión democráticos posibilita el empoderamiento de actores y sectores muchas veces invisibilizados en los procesos de producción de hábitat.

Consecuentemente, en la búsqueda de dilucidar insumos emergentes para la generación de un hábitat sustentable y más justo cognitivamente, se plantea como estrategia metodológica el estudio del caso a partir de tres categorías analíticas elaboradas en tal sentido:

a) Contexto de aplicación (valoración de la capacidad instalada). Esta categoría analítica hace referencia a la producción de conocimiento y su relación con la utilidad social del mismo. Esta intención, de producir conocimiento que sea útil para alguien, ya sea el sector productivo, los gobiernos locales o la sociedad en general, está plasmada de manera relevante desde el inicio de las intervenciones o investigaciones. Por tanto, esta categoría permite analizar cuál es el contexto de aplicación del caso, cuáles son las capacidades valoradas y rescatadas para la producción de hábitat y a qué sectores específicos de la sociedad se beneficia.

b) Encuentros productivos: Co-construcción del conocimiento (producción de conocimiento colectivo). Esta categoría analítica es una premisa investigativa que el equipo de investigación viene desarrollando desde hace varios años en los procesos de producción de hábitat. La Co-construcción de conocimiento adscribe a un tipo de trabajo colectivo, donde los saberes de los diversos actores son incorporados a los procesos productivos. Permite analizar qué tipo de conocimientos circulan en esos espacios denominados "encuentros productivos", cómo se construyen dichos espacios y qué significado se le otorga a la propiedad intelectual de los resultados obtenidos.

c) Alianzas inter-actorales/inter-sectoriales (gestión democrática). Como premisa investigativa, esta categoría asume a la gestión como una activa articulación intersectorial, cuya estrategia radica en convocar en la resolución de la problemática del hábitat a la mayor cantidad de sectores relevantes para la definición de políticas públicas que generen beneficios distribuidos. Esta permite analizar los acuerdos surgidos a partir de este tipo de gestión, cómo se comparte la información, cómo convergen los sentidos e intereses de cada sector participante y cómo se toman las decisiones.

Caracterización del caso de estudio: La experiencia toma lugar en la ciudad de Concordia (provincia de Entre Ríos), ubicada en el litoral argentino (Figura 1). Como segunda ciudad más grande de dicha provincia, se caracteriza por la relevancia de la actividad forestal, ya que presenta la mayor superficie de árboles de Eucalyptus Grandis. Desde el año 2010, un grupo de investigadores de CONICET ha implementado en esta localidad (a través de diversos proyectos de investigación) una Red Inter-actoral, con el objetivo de llevar adelante desarrollos tecnológicos en madera, poniendo en valor desde sus inicios la matriz productiva local (Peyloubet, 2017) (Figura 2). En ese contexto de aplicación y bajo un proceso de innovación cognitiva, se ha desarrollado concretamente una tecnología constructiva maderera que, por su configuración de componentes, posee un diseño flexible y adaptable a diferentes usos (vivienda, equipamiento urbano, plantas de reciclado, etc.). Mediante dicha propuesta, se intenta traccionar la producción foresto- industrial de la región y localidad, con el fin de fomentar el potencial laboral en el sector, haciendo uso eficiente del recurso madera y diversificando el mismo para alcanzar niveles de rentabilidad mayores, y así generar sobre la cadena de valor una plusvalía en el uso de la materia prima a partir del diseño de producto (sistema constructivo). De igual modo, se advierte que la propuesta involucra a pequeños productores que forman parte de la economía vulnerable de la localidad, cuya inserción en el mercado requiere de un apalancamiento del Estado.

\section{DISCUSIÓN Y RESULTADOS}

\section{A) CONTEXTO DE APLICACIÓN: VALORACIÓN DE LA CAPACIDAD INSTALADA}

La experiencia en Concordia se caracteriza por un modo de producción de conocimiento alternativo a los mecanismos tradicionales o hegemónicos. El equipo de investigación de CONICET ha venido desarrollando en otras localidades 

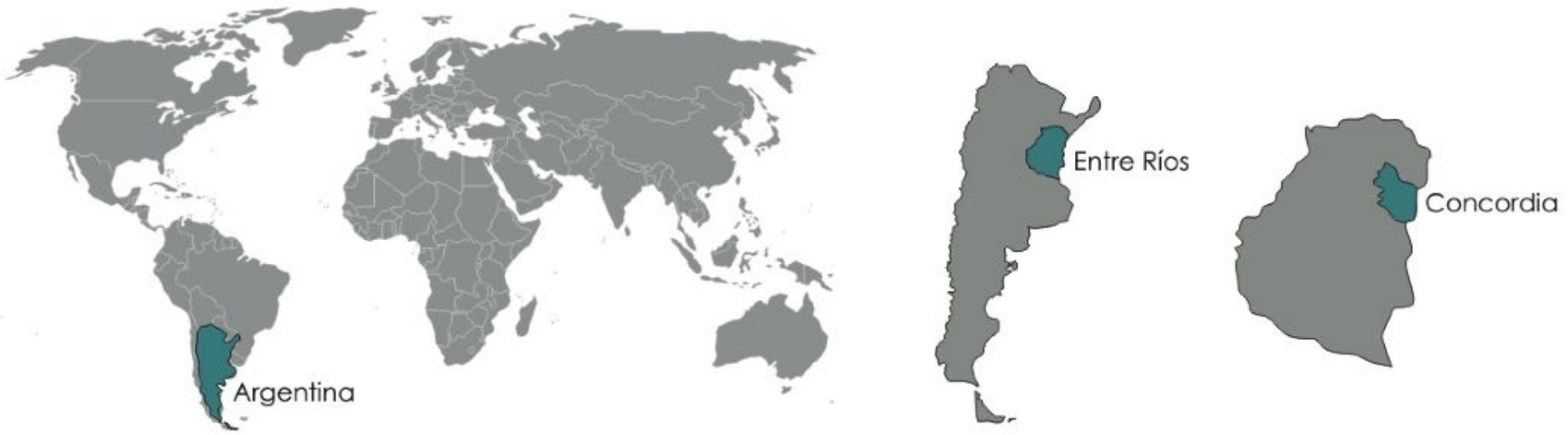

Figura 1. Ubicación geográfica. Fuente: Elaboración de la autora.
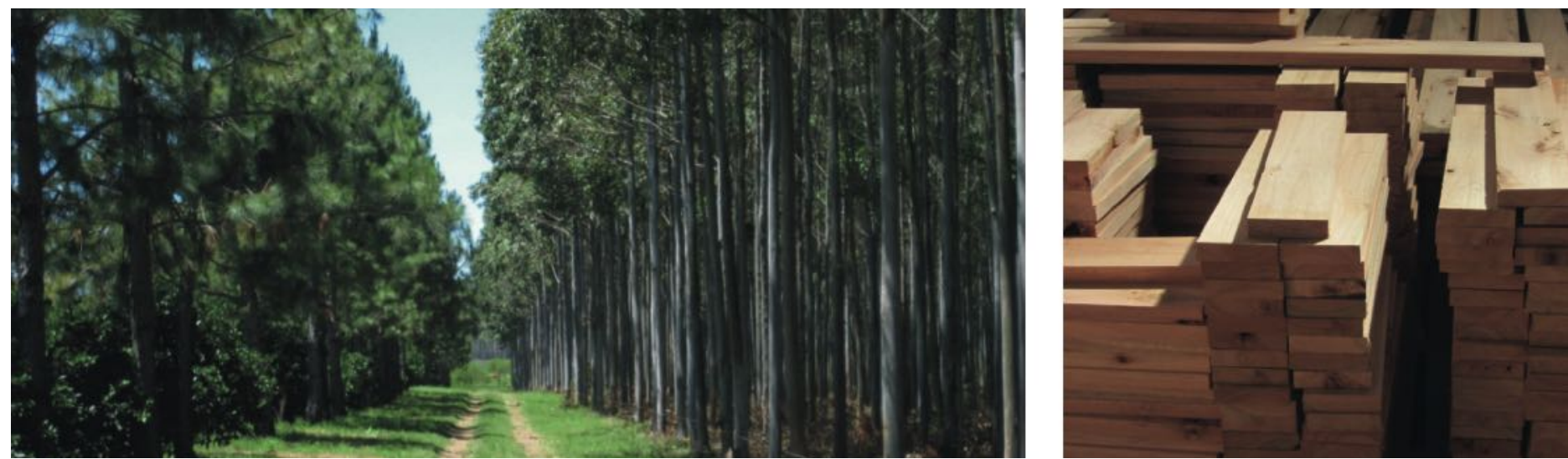

Figura 2. Producción forestal. Madera aserrada Eucalytus Grandis. Fuente: Imagen extraída de PROCODAS (2011) y PAD-ANPCYT (2017).

procesos de producción de hábitat donde la resolución de la problemática se ha generado a partir de circuitos productivos inter-actorales y no desde la concepción del artefacto, es decir, la vivienda. Ese modo alternativo de abordar la producción de hábitatllamó el interés de funcionarios municipales de Concordia y el año 2010 el equipo de investigación fue convocado por la Secretaría de Producción y Trabajo para trabajar en proyectos que dinamizaran las cadenas productivas locales del ámbito forestal, la producción de vivienda y generación de trabajo para pequeños productores. En ese contexto, Concordia contaba con una importante capacidad instalada: bosques de Eucalyptus, aserraderos y una Asociación de Carpinteros.

Frente a esta sub-utilización de recursos y conocimientos locales, la propuesta de intervención en el territorio se desarrolló bajo las siguientes premisas de diseño: a) Reconocer y valorar la matriz productiva local: se recorrieron aserraderos y bosques, con objetivo de poner en relevancia la capacidad instalada (Figura 3).

b) En base a dicho reconocimiento y valoración, utilizar el mayor porcentaje posible de madera local (Eucalyptus Grandis) para la construcción de componentes: esto permitiría re-diversificar y re-significar en la población el uso que se hace del recurso maderable en la localidad².

c) A partir de una demanda local, se decide diseñar como prototipo piloto un salón de usos múltiples (SUM), con un sistema constructivo realizado con predominancia de tablas de Eucalyptus Grandis de 1"x4", reconocidas estas como la matriz productiva local (Figura 4).

[2] En este punto, es clave aclarar que la elección de decidir por utilizar la matriz productiva local (tablas de madera de sección muy pequeña), para re-definir el uso de la madera de Eucalyptus Grandis, generó el desarrollo de un diseño estructural particular, que fuera capaz de responder prioritariamente a la solicitud de esfuerzos de viento. En tal sentido, fue necesario someter dicho diseño a rigurosos cálculos estructurales, quedando conformada una estructura de pórticos (paneles de cerramientos), columnas y una estructura de techo. Como resultado de dichos cálculos, se determinaron las dimensiones de los elementos del sistema, la colocación de diagonales para generar rigidez a la estructura, la cantidad y tipo necesario de vinculaciones metálicas, etc. 


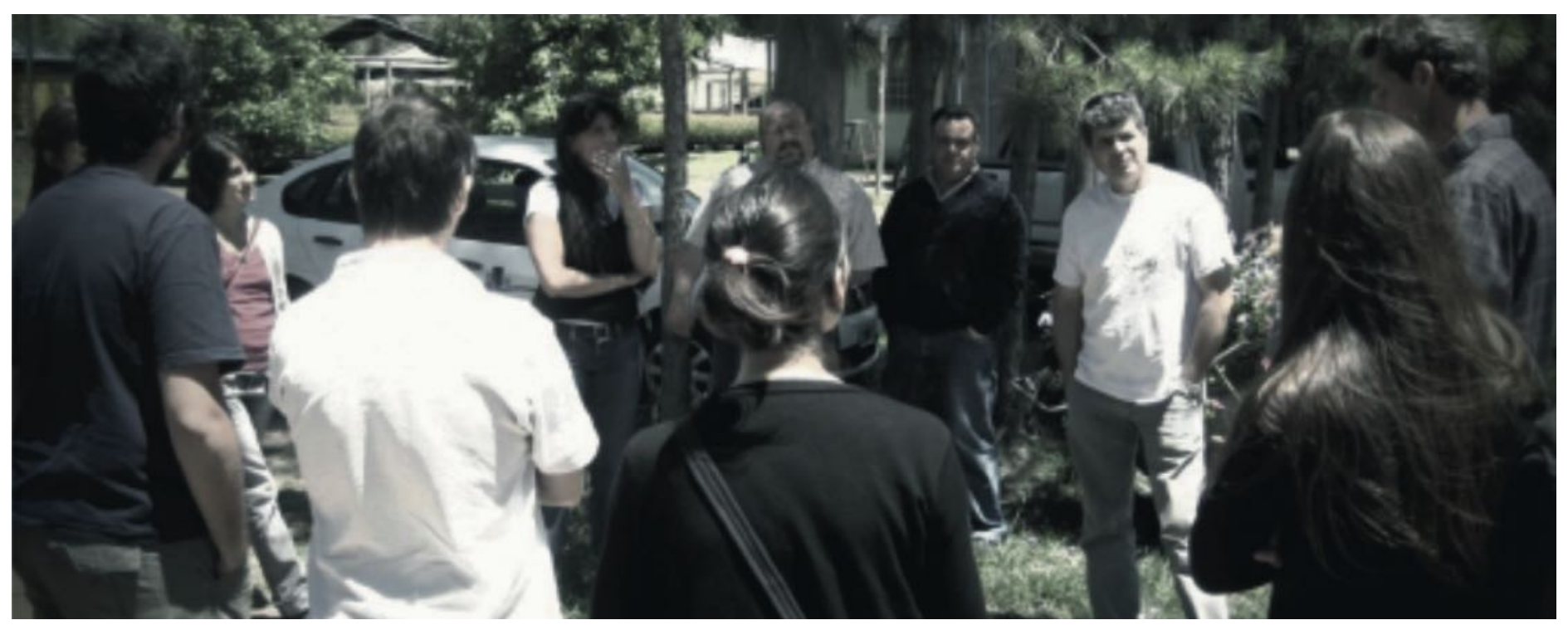

Figura 3. Recorrido por producciones forestales locales. Fuente: Imágenes extraídas de DETEM (2012).
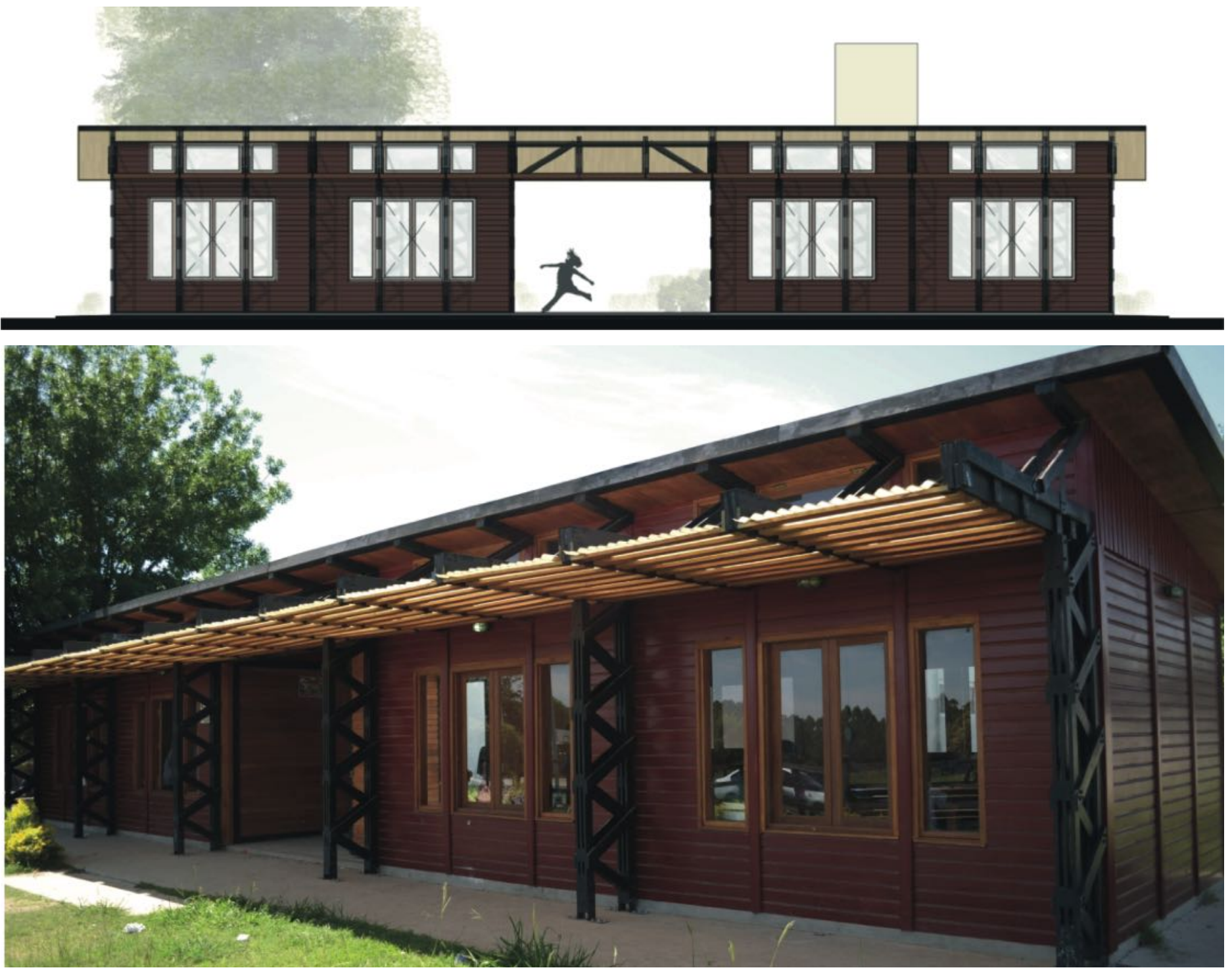

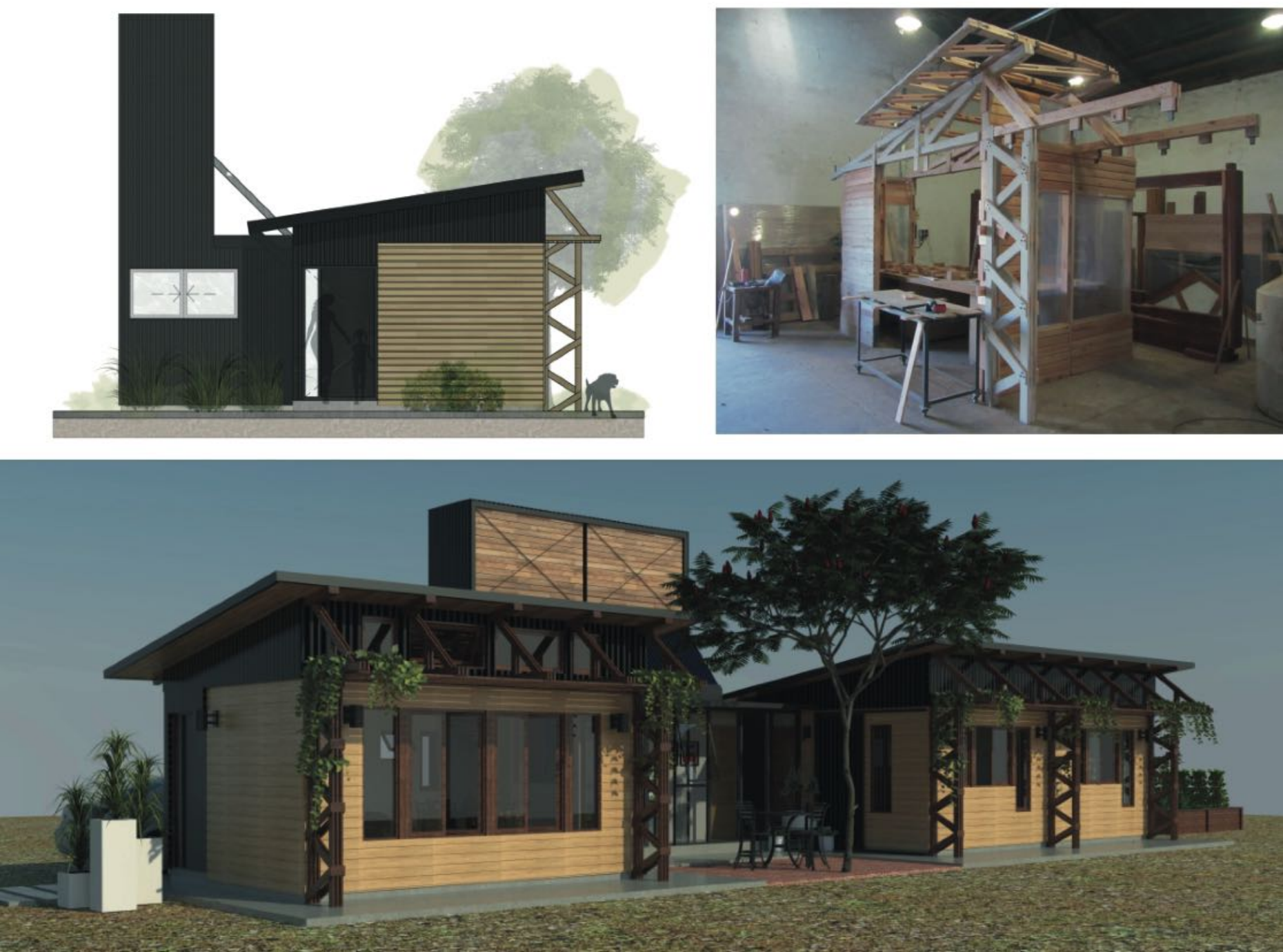

Figura 5. Sistema constructivo de componentes prefabricados que se adapta a distintas tipologías como vivienda o equipamientos barriales. Fuente: Imágenes extraídas de PID 0079 (2016).

d) Diseñar un desarrollo tecnológico de producto flexible capaza de adaptarse a diferentes requerimientos y usos: viviendas o equipamientos barriales (Figura 5).

Asimismo, la elección de un recurso natural renovable como la madera de Eucalyptus para la construcción implicó explorar provechosamente varias dimensiones del problema: en términos ambientales, se estaba utilizando un recurso maderero de bosque de crecimiento rápido que, al ser seleccionado como material para la construcción, contaba con significativas ventajas comparativas en relación con otros materiales de construcción, entre las que destacan las siguientes:

- La producción de madera actúa como almacén de carbono purificando el aire y contribuyendo a la reducción del efecto invernadero;

- Los procesos de producción y transformación de la madera consumen menos energía que los procesos productivos de otros materiales;
- Mucha de la energía que se consume proviene de sus propios residuos, por ende, la industrialización de la madera incide positivamente en la reducción de la demanda de combustibles sólidos;

- Se puede utilizar las cenizas de madera como fertilizantes para el campo.

Las decisiones y elecciones que se tomaron de manera colectiva en el inicio del proyecto de investigación buscaron respaldarlosintereses del sectorproductivolocaly responder a la demanda del sector gubernamental (municipio local), cuyo propósito fue re-dinamizar la economía local. Es decir, este constituye una propuesta de producción de hábitat que responde a las necesidades locales, y no al revés, como cuando la comunidad es la que tiene que ajustarse a la nueva tecnología introducida (proceso dominante en la construcción de tecnologías). Siguiendo aquí a Herrera (2010), es muy frecuente la detección de propuestas y desarrollos tecnológicos focalizados en la innovación del producto que, por lo general, desestiman, de manera 


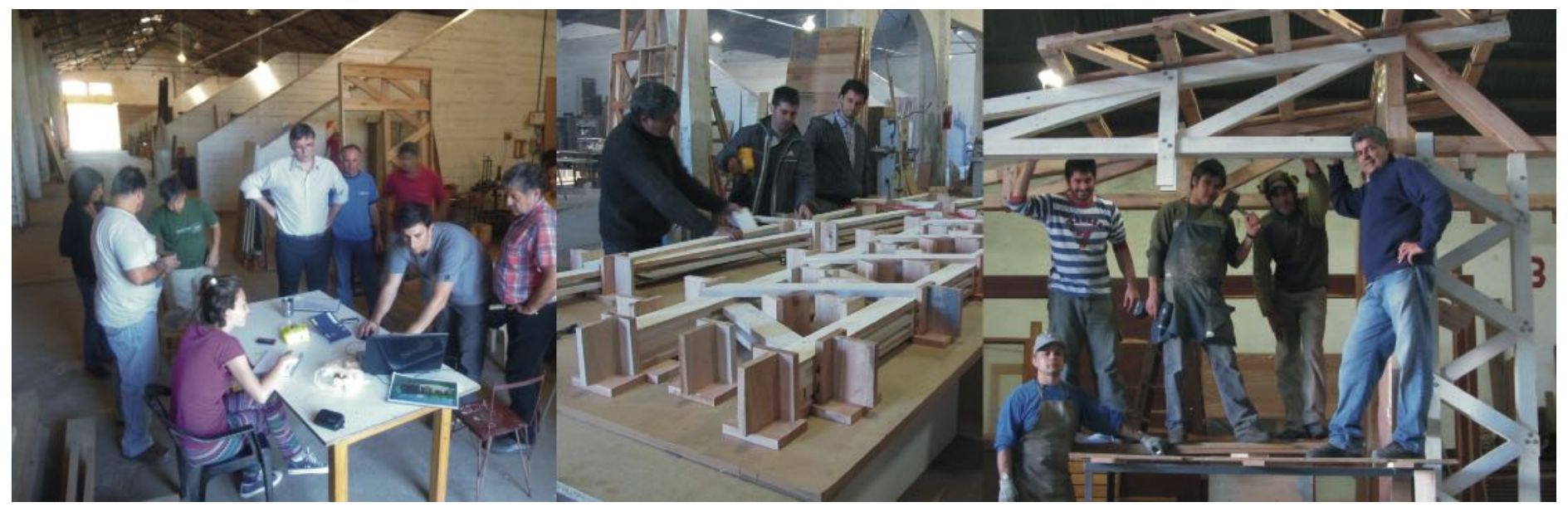

Figura 6. Lugar potencial de producción de conocimiento, llamado como Encuentros Productivos. Fuente: Imágenes extraídas de PID 0079 (2016).

previa, todas las condiciones sociales y técnicas que los actores locales pueden proveer a la construcción de dicha tecnología. El hecho de que el proyecto revalorizara el oficio y la capacidad local en la ciudad de Concordia hizo que los actores pudieran trabajar, desde su identidad; básicamente, desde lo que son y saben hacer, creando un sujeto social que puede modificar y apropiarse del producto tecnológico en base a su experiencia en el oficio (generación de trabajo autónomo). En esa dirección, la búsqueda de valor agregado a un recurso sub-utilizado y el reconocimiento de la capacidad instalada en la localidad constituyen unos de los elementos que configuran las normas cognitivas y sociales que gobiernan el proceso de producción de conocimiento en la experiencia en Concordia.

\section{B) ENCUENTROS PRODUCTIVOS: CO-CONSTRUCCIÓN DEL CONOCIMIENTO (PRODUCCIÓN DE CONOCIMIENTO COLECTIVO)}

La propuesta metodológica de abordaje de producción de hábitat que plantea esta experiencia adscribe, como se ha sostenido, a un tipo de trabajo colectivo donde los saberes de los diversos actores son incorporados a los procesos productivos, en una integración que permite abrir las cajas negras del saber experto de algunos y reivindicar el saber técnico y experiencial de otros. Esta metodología fue denominada por el equipo de investigación como "Coconstrucción del conocimiento". Según Peyloubet (2018), la idea fundamental en propuestas de trabajo colaborativo como ésta se asienta en la complementariedad, que desplaza a la competitividad, en un accionar asociativo, donde la propiedad intelectual es compartida. De esta manera, el desarrollo de la tecnología constructiva, es decir de producto, se crea en espacios de interacción llamados "encuentros productivos" donde, de manera grupal y mediante una participación respetuosa, se van plasmando ideas, saberes y decisiones técnicas sobre la tecnología en cuestión. Para los actores participantes -carpinteros, investigadores, técnicos municipales, cooperativistas de trabajo-, estos encuentros son espacios donde el saber y el conocimiento circulan solidariamente y donde todos enseñan y todos aprenden, dándose como resultado una construcción colectiva de la tecnología. En ese sentido, lo paradigmático y beneficioso de la experiencia es que el desarrollo tecnológico no llegó a la localidad como un sistema cerrado, a modo de caja negra y junto con un manual de uso, sino todo lo contrario. La predisposición de los técnicos investigadores al intercambio de conocimiento estuvo presente al llegar a la Asociación de Carpinteros de Concordia. La decisión de no transferir tecnologías por un acercamiento dialógico con los actores intervinientes le proporcionó de manera implícita a la tecnología cualidades políticas, tales como:

- La posibilidad de los actores intervinientes de sentirse parte del desarrollo de la tecnología;

- La posibilidad de cambio, ajustes y nuevas ideas a partir del intercambio,

- El enriquecimiento de todos los actores intervinientes, incluso el propio sector académico, gracias al intercambio de distintos saberes que generó nuevos aprendizajes;

- Apertura a los problemas y a la realidad local, la cual hizo posible que la tecnología fuera lo más adecuada social y técnicamente a dicho contexto.

El fuerte reconocimiento de lo local, y el uso de una metodología de trabajo de Encuentros Productivos, promovió otra instancia de transición en los modos de operar del sector de CyT. Una vez montado y construido el SUM, y luego del inicio de la construcción de la primera vivienda como prototipo, se adviertió de manera colectiva la necesidad de gestionar un Certificado de Aptitud Técnica ${ }^{3}$ (CAT) de tal sistema. 


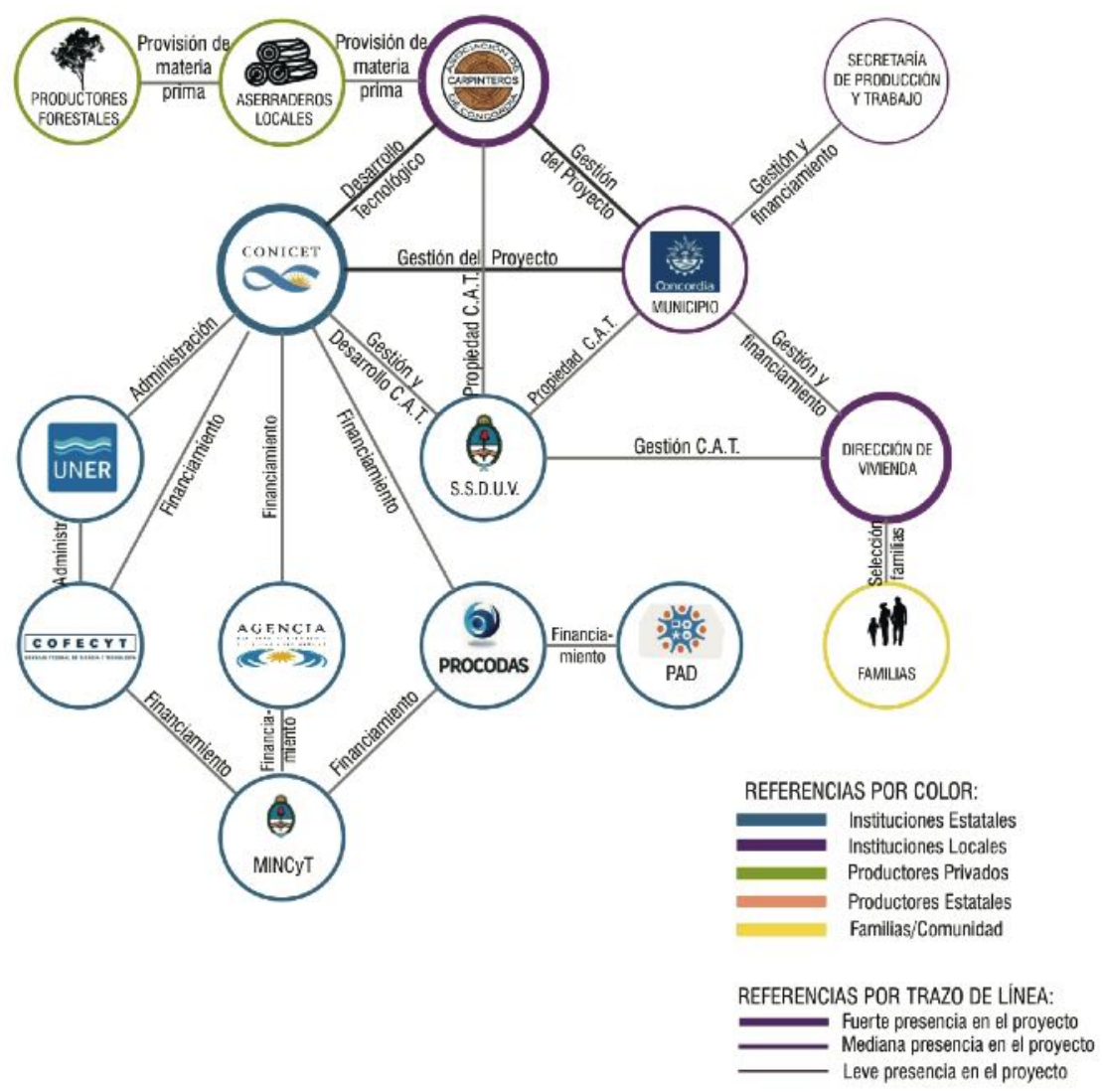

Figura 7. Red interactoral de la experiencia en Concordia. Fuente: https://coconstrucciondelconocimiento.wordpress.com

Para el equipo de investigación, el sistema constructivo al que se había llegado era el resultado de la integración de ideas, saberes y soluciones que la comunidad había usado tradicionalmente en la construcción de viviendas de madera (producto), así como también de los aspectos de gestión y comercialización de recursos locales (proceso). Por ello, se decidió dejarlo en manos del actor municipal y la Asociación de Carpinteros. Una tecnología cuya resolución final fue producto de una complementariedad de saberes diversos no podía estar limitada por patentes que involucraran derechos lucrativos para los supuestos desarrolladores, tecnólogos, investigadores, entre otros participantes. Es decir, no hay un actor, un héroe detrás de esa tecnología, sino que hay varios actores que pueden ir cambiando y que, además, pueden ir adaptando continuamente la tecnología, sin que esta dependa de alguien en particular o exclusivamente. En este marco, la decisión colectiva acerca de solicitar el CAT del desarrollo tecnológico significó para la experiencia una manera de descentralizar la política convencional de vivienda y reemplazarla por una nueva forma de gestionar la política habitacional desde un municipio local.

\section{C) ALIANZAS INTER-ACTORALES/INTER-SECTORIALES (GESTIÓN DEMOCRÁTICA)}

Otro elemento clave de innovación tecnológica en la experiencia aquí compartida lo constituye la articulación interactoral e intersectorial como norma cognitiva y social de producción de conocimiento. El propósito de dicha estrategia radica en convocar a la resolución de la problemática del hábitat a la mayor cantidad de sectores relevantes que puedan contribuir a la definición de políticas públicas orientadas a generar beneficios distribuidos. En esa línea, para el equipo de investigación los sectores que deben estar presentes son:

1. Sector del Estado: en cuanto guardianes del bien común; 2. Sector productivo: emprendedores en la cadena de valor; 3. Sector cognitivo: mediadores de los saberes diversos.

En la experiencia, la mesa de participación estuvo integrada por los siguientes sectores: representantes del municipio (Instituto de Tierras y Viviendas, Sub Secretaría de Desarrollo Económico), representantes del sector productivo (Productores Forestales, Aserraderos, Cooperativas de Trabajo, Asociaciones de Carpinteros) y representantes del sector cognitivo (Universidades, Centros y Agencias de Investigación); quienes en sucesivas deliberaciones fueron construyendo acuerdos para innovar en los procesos productivos que afectan a los tres sectores, procurando promover el trabajo con autonomía y la coresolución de problemáticas locales (Figura 7).

Los instrumentos de financiamientos que sostienen este tipo de tecnología provienen del sector de Ciencia y Tecnología, ya que el grupo promotor y mediador está 

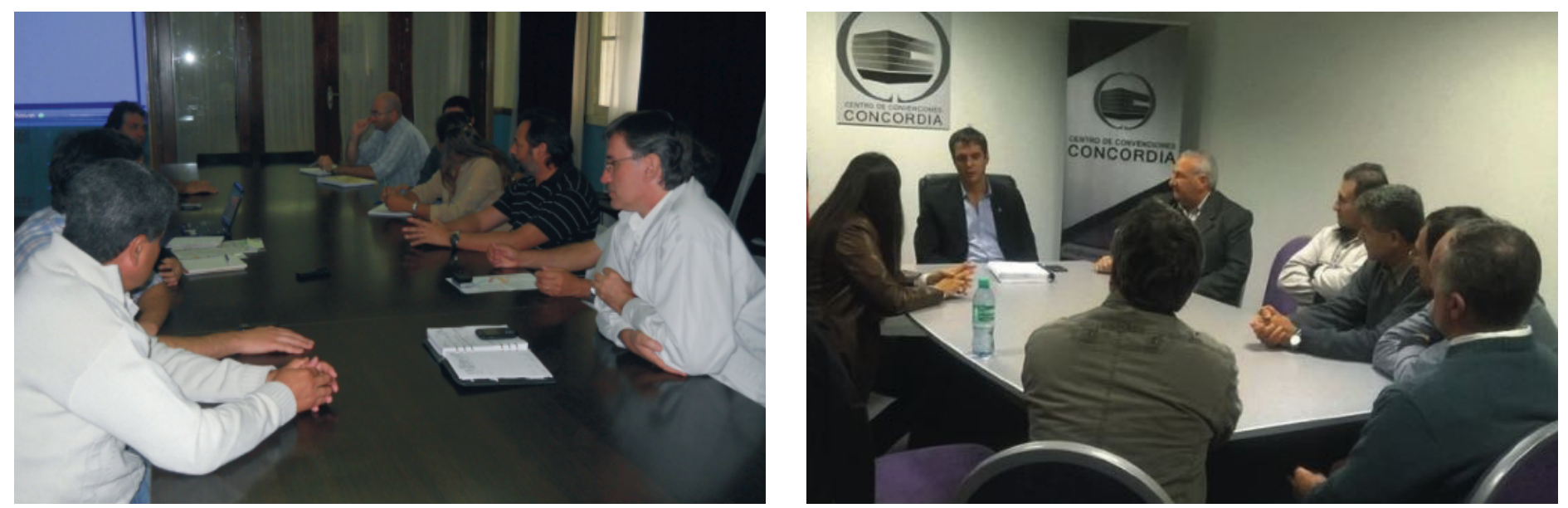

Figura 8. Alianzas inter-sectoriales / articulación de políticas. Fuente: Imágenes extraídas de PROCODAS (2011; 2017).

conformado por investigadores (sector cognitivo). Cabe aclarar que la propuesta de la Red interactoral ha contado durante los casi diez años de su existencia con el apoyo y aprobación de proyectos de financiamiento de dicho sector, ya que los aspectos clave de esta propuesta cobran relevancia por ser instrumentos de una política científica-tecnológica establecida mediante el Plan Argentina Innovadora 2020. La iniciativa contó con cinco proyectos de investigación financiados por la Agencia Nacional de Promoción Científica y Tecnológica. La continuidad de los instrumentos de financiamiento en la experiencia fue clave, ya que permitieron avanzar con los objetivos planteados y engendró en lo actores participantes la motivación de seguir formando parte de la Red interactoral.

La premisa metodológica e ideológica de generar un modelo de gestión democrático fue esencial para el proyecto. Las sucesivas reuniones con cada representante de los sectores permitieron que allí circulara una exhaustiva información, una profunda comprensión y una democrática decisión de los aspectos y cuestiones que tienen que ver con el desarrollo de la tecnología (Peyloubet, 2018).

Un aspecto a destacar fue que las distintas reuniones en el municipio con el intendente y funcionarios contaron siempre con la presencia de los productores del desarrollo tecnológico (Asociación de Carpinteros y Cooperativas). En ese sentido, el proyecto siempre buscó el empoderamiento de sectores, muchas veces silenciados a la hora de tomar decisiones. Esta estrategia dio paso, hacia el interior de los grupos o asociaciones, a una gran disponibilidad de información y de propuestas para poder tomar el control sobre su trabajo y advertir problemas, soluciones y nuevas oportunidades relacionadas con sus modos de trabajo. Se generó así un proceso democrático donde todos se posicionaron como co-partícipes y co-responsables del o los proyectos en curso. Este modo de gestionar la tecnología, que hace referencia a la presencia de todos los actores, sin establecer jerarquías, fomentó la construcción de una red de relaciones externas a nivel nacional como regional, donde las organizaciones, y particularmente, la Asociación de Carpinteros, lograron establecer contactos e información relevante, como así también propuestas de nuevos trabajos. El vínculo y las alianzas generadas en el marco de la Red permitieron un nuevo modo de participación en la resolución de problemáticas socio-productivas, donde (en este caso) el Estado (gobierno municipal) representó los intereses de los grupos o economías más pequeñas, originando oportunidades de trabajo y acceso a recursos, en el marco de decisiones colectivas y democráticas (Figura 8). De esta manera, la Asociación de Carpinteros accedió a y gestionó nuevos beneficios por fuera del proyecto en estudio, entre los que se cuentan:

1) Capitalización en infraestructura: a través del Ministerio de Desarrollo Social de la Nación obtuvieron una machimbradora ${ }^{4}$;

2) Venta de componentes de madera para el municipio local (puertas y refugios para guardavidas en madera, para colocar en las orillas del río Paraná);

3) Nuevas Estaciones Saludables en madera para la localidad;

4) Capacitaciones en el marco de proyectos del Ministerio de Trabajo y Producción;

5) Convenio con el Ministerio de Desarrollo Social de la Nación para la producción de componentes estructurales de techo (madera), para una planta de tratamientos de residuos en la localidad de Concordia. 


\section{CONCLUSIONES}

La propuesta expuesta aquí viene realizándose con éxito desde el año 2010, y ha sido posible, principalmente -a partir de lo que los propios participantes atribuyen-, a la confianza construida entre los diferentes miembros que conforman la Red socio-productiva. Por un lado, la convicción teórica e ideológica del grupo de investigación es plasmada año tras año en la formulación y presentación de nuevos proyectos ante el Ministerio de Ciencia y Tecnología de la Nación; institución que aprueba y continúa apoyando este tipo de desarrollos tecnológicos, mediante sus diferentes líneas de financiamiento. Por otro lado, los carpinteros de la Asociación, como el actor productivo principal, continúan apostando en generar nuevos caminos y nuevos procesos que promuevan beneficios distribuidos en su localidad. Y un municipio, que, si bien en los inicios del proyecto estuvo muy presente y luego no tanto, nunca dejó de apalancar y apoyar la propuesta investigativa.

Frente a ese escenario, se pueden destacar, a modo de conclusión, tres aspectos claves de innovación tecnológica que la experiencia en Concordia llevó adelante para el abordaje de problemáticas relacionadas con el hábitat:

-Desarrollos tecnológicos socio-productivos locales: la iniciativa se basa en un abordaje que permite comprender y aprovechar en plenitud las oportunidades que ofrece la trama productiva local, desarrollando propuestas que persiguen potenciar y diversificar nuevas formas y procesos productivos para generar dinámicas económicas re-distributivas. En el caso de la experiencia en estudio, el equipo de investigación adviertió una sub-utilización de los recursos naturales (renovable-madera), como también de las capacidades productivas locales, siendo estas consideradas como nuevas oportunidades emergentes.

-Desarrollos Tecnológicos Colaborativos y Asociativos: la metodología que implementa la propuesta adscribe a un tipo de trabajo colectivo, donde los saberes de los diversos actores son incorporados a los procesos productivos, en una integración que consigue abrir las "cajas negras2 del saber consuetudinario de algunos y potenciar el saber técnico y experiencial de otros. La propuesta de la coconstrucción constituye uno de los elementos investigativos más relevantes del equipo de CONICET, el cual obliga a concebir a los desarrollos tecnológicos como un patrimonio cognitivo de un conjunto de emprendedores que se organizan en una economía social basada en la distribución de la renta y la inclusión socio-productiva, promoviendo un mercado más solidario. Esa co-construcción se manifiesta fundamentalmente en lo que el equipo de investigación llama Encuentros Productivos donde, de manera grupal y en una participación respetuosa, se acuerdan las ideas y decisiones técnicas que darán a luz el producto tecnológico.

-Desarrollos Tecnológicos Inter-sectoriales: el abordaje para la construcción de un hábitat más justo y sostenible se da desde la articulación de diversos actores y sectores que, desde sus diferentes posiciones e intereses promueven acuerdos surgidos de una exhaustiva información y una democrática decisión. Para la propuesta en estudio, la presencia de actores del Estado (guardianes del bien común), actores productivos (emprendedores en la cadena de valor) y actores cognitivos (mediadores de los saberes diversos) se constituye en un aspecto relevante para la promoción de nuevas políticas públicas en el campo de producción de hábitat.

Así, las normas cognitivas y sociales que gobiernan el proceso de diseño y desarrollo de tecnología en la experiencia se basan en aspectos, tales como: a) desarrollos tecnológicos que impacten lo menos posible en el ambiente (utilización de recurso renovable); b) desarrollos que apalanquen economías pequeñas y vulnerables; c) desarrollos que promuevan formas de producción asociativas y cooperativas; d) desarrollos que generen distribución justa de la renta; $y, e)$ desarrollos que co-diseñen un producto de propiedad colectiva.

En consecuencia, pensar los procesos de desarrollo tecnológico desde este enfoque representa una invitación a abordar la problemática del hábitat a partir de una construcción sistémica, ya que la dimensión, social, cultural, económica y ambiental, se encuentra en un mismo nivel de jerarquía y el producto tecnológico constituye una excusa para dinamizar y democratizar procesos tecnológicos. Asimismo, permite aproximarse a la problemática desde una perspectiva política, asumiendo que en la tecnología se concretizan disposiciones de poder, inscriptas en los desarrollos tecnológicos desde las premisas con que fueron diseñados. Este abordaje proporciona además la oportunidad de tomar conciencia sobre la importancia de la forma en que las decisiones y elecciones técnicas influyen en los procesos tecnológicos y en la necesidad de una conciencia crítica acerca de los modos convencionales de hacer tecnología. Esto conduce a asumir la relevancia de instancias reflexivas, capaces de ser llevadas adelante por gestores, técnicos, profesionales, académicos, etc., que den como resultado el desarrollo de propuestas en territorios con miras a aumentar las opciones de proceder a otras operaciones alternativas en el campo del hábitat.

En base a los resultados obtenidos, se puede señalar que cuando se enfrentan problemáticas socio-productivas de una manera conjunta, participativa y desde su génesis, se estaría desarrollando una tecnología de cualidades políticas con normas cognitivas y sociales de producción de conocimiento diferenciadas. La posibilidad de generar un proceso tecnológico donde la problemática sea coconstruida, y de manera interactoral, permite la circulación y puesta en común de conocimientos y soluciones locales que son propias de los sectores relevantes de un territorio determinado.

Desde ese punto de vista, la experiencia en Concordia representa una oportunidad para sumergirse en un proceso complejo de deconstrucción para luego reconstruir una 
tecnología que permita resolver problemáticas socioproductivas a partir de un abordaje tecnológico socioproductivo, solidario, cooperativo e inter-sectorial. Este trabajo, en definitiva, se constituye en una oportunidad de continuar preguntándose hacia dónde y cómo debiera dirigirse el desarrollo de una tecnología capaz de configurar un hábitat más justo y sustentable.

\section{AGRADECIMIENTOS}

La presente investigación viene siendo desarrollada y financiada en el marco de los siguientes proyectos de investigación:

-PICT N ${ }^{\circ}$ 1737: Innovación para la gestión de Tecnología Social en el campo del hábitat.

(Proyectos de Investigación científica y tecnológica con fondos provenientes del FONCYT (AGENCIA NACIONAL DE PROMOCIÓN CIENTÍFICA Y TECNOLÓGICA);

-PFIP 2018: "Fortalecimiento de la producción maderera local a partir de la generación de una línea de desarrollo, producción y montaje de componentes de madera (Eucalyptus Grandis) para vivienda y su mobiliario, que consolide a la Asociación de Carpinteros de Concordia como sede de producción, transferencia y capacitación regional". Proyectos Federales de Innovación Productiva con fondos provenientes del COFECYT (CONSEJO FEDERAL DE CIENCIA Y TECNOLOGÍA).

\section{REFERENCIAS BIBLIOGRÁFICAS}

Albornoz, M. (2013). Innovación, equidad y desarrollo latinoamericano. Isegoría. Revista de Filosofía Moral y Política, (48), 111-125.

Dagnino, R. (2008). Neutralidade da ciencia e determinismo tecnológico. Um debate sobre a tecnociencia. $1^{\circ}$ edición. Brasil, Campinas: Editorial Unicamp.

DETEM - ANPCYT. (2012). Circuito productivo interactoral a partir de una tecnología social para la producción de vivienda de madera. Caso: Concordia, Provincia de Entre Ríos.

Feenberg, A. (2006). Del esencialismo al Constructivismo: la Filosofía de la tecnología en la encrucijada. Recuperado de http://www.sfu.ca/ andrewf/translations.html

Fresoli, M., Smith, A., Thomas, H. y Bortz, G. (2016). De las Tecnologías Apropiadas a las Tecnologías Sociales: algunos dilemas persistentes de los movimientos de innovación de base para la construcción de futuros socialmente justos. En Thomas, H. y Santos, G. (Eds.), Tecnologías para incluir. Ocho análisis socio-técnicos orientados al diseño estratégico de artefactos y normativas. Argentina. Quilmes: Editorial Instituto de Estudios Sobre la Ciencia y la Tecnología, pp. 49-93.
Giuliano, G. (2012). Comentarios sobre el texto: Ciencia, Tecnología y Democracia. En: I Encuentro Internacional Culturas Científicas y Alternativas Tecnológicas (Buenos Aires, 2012). Recuperado de http://es.scribd.com/doc/20694868/ Comentarios-de-Gustavo-Giuliano-sobre-el-trabajo-de-AndrewFeenberg-www-eiccat-com-ar

Guba, E. y Lincoln, Y. (1994). Paradigmas en competencia en la investigación cualitativa. En Denzin y Lincoln (Eds.), Handbook of Qualitative Research. Thousand Oaks, California, Sage.

Herrera, A. (2010). La generación de tecnologías en las zonas rurales. En Dagnino, R. (Ed.), Tecnologia social. Ferramenta para construer outra sociedade. Campinas. Brasil: Editorial Komedi, pp. 23-51.

Kazez, R. (2009). Los estudios de caso y el problema de la selección de la muestra. Aporte del sistema de matrices de datos. Subjetividad y Procesos cognitivos, 13(1), 71-89.

OEI (2013). Ciencia, Tecnología e Innovación para el desarrollo y la cohesión social. Madrid, España: Organización de Estados Iberoamericanos.

PAD-ANPCYT. (2017). Co-diseño de equipamiento para instituciones públicas a partir del recurso maderero local (Eucalyptus Grandis). Constitución de Consorcios TecnoProductivos, público-privados, en el marco de la Economía Social.

Peyloubet, P. (2017). Animarse a habitar. Buenos Aires: Editorial Diseño.

Peyloubet, P. (2018). Convidar Tecnología. Una propuesta a partir de la Co-construcción. Buenos Aires: Editorial Diseño.

PID 0079-ANPCYT. (2016). Producción a escala de viviendas de madera, en el marco de un circuito productivo interactoral a partir del uso de una tecnología social. Caso: Concordia, provincia de Entre Ríos.

PROCODAS - ANPCYT (2011). Tecnología para la inclusión social en el marco del desarrollo local. Caso: Concordia, provincia de Entre Ríos.

PROCODAS -ANPCYT. (2017). Promoción y capacitación en tecnologías constructivas de eucalipto que dinamicen la economía social de la región, a partir del recurso foresto-industrial emergente, para el fortalecimiento del desarrollo productivo, en el marco del consorcio intermunicipal del departamento de Concordia, Entre Ríos.

Santos, B. (2009). Una epistemología del Sur: la reinvención del conocimiento y la emancipación social. México: Editorial CLACSO.

Servicio Nacional de Sanidad y Calidad Agroalimentaria (SENASA). (2014). Bosques argentinos, actividad forestal y economías regionales. Recuperado de http://www.senasa.gob. ar/senasa-comunica/infografias/bosques-argentinos-actividadforestal-y-economias-regionales

Thomas, H. y Becerra, L. (2014). Sistemas tecnológicos para el desarrollo inclusivo sustentable. Revista Voces en el Fénix, (37), 120-129 
Tula Molina, F. y Giuliano, G. (2015). La teoría crítica de la tecnología: revisión de conceptos. Revista Redes, 21(40), 179214.

Tula Molina, F. y Giuliano, G. (2017). El riesgo de que todo funcione. Buenos Aires: Nueva librería.

Valderrama, A. (2004). Teoría y Crítica de la Construcción Social de la Tecnología. Revista Colombiana de Sociología, (23), 217233.

Vasilachis de Gialdino, I. (2006). Estrategias de investigación cualitativa. España, Barcelona: Editorial Gedisa. 